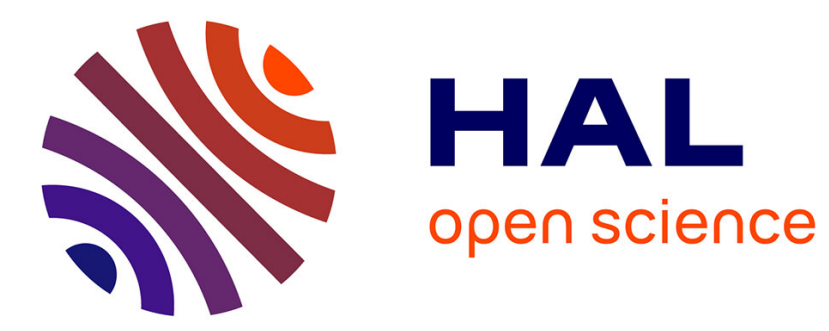

\title{
La négociation du temps de travail: l'emploi en jeu
}

Marie-Laure Morin, Gilbert de Terssac, Jens Thoemmes

\section{To cite this version:}

Marie-Laure Morin, Gilbert de Terssac, Jens Thoemmes. La négociation du temps de travail: l'emploi en jeu. Sociologie du Travail, 1998, 40 (2), pp.191-207. hal-00937155v2

\section{HAL Id: hal-00937155 \\ https://hal.science/hal-00937155v2}

Submitted on 5 Aug 2014

HAL is a multi-disciplinary open access archive for the deposit and dissemination of scientific research documents, whether they are published or not. The documents may come from teaching and research institutions in France or abroad, or from public or private research centers.
L'archive ouverte pluridisciplinaire $\mathbf{H A L}$, est destinée au dépôt et à la diffusion de documents scientifiques de niveau recherche, publiés ou non, émanant des établissements d'enseignement et de recherche français ou étrangers, des laboratoires publics ou privés. 
Morin M.-L., De TerssaC G., ThOemmes J., 1998, « La négociation du temps de travail: l'emploi en jeu », Sociologie du Travail, 40, 2, p. 191-207.

\section{LA NEGOCIATION DU TEMPS DE TRAVAIL : L'EMPLOI EN JEU}

\section{M.L. Morin, G de Terssac, J. Thoemmes*}

En France, les enjeux liés à la transformation de la durée et de l'organisation du temps de travail ont longtemps été placés sous le signe de l'amélioration des conditions de travail et du temps libre. Aujourd'hui, le paysage n'est plus le même puisque le temps de travail est devenu un levier majeur de la politique de l'emploi (Freyssinet, 1997 ; Thoemmes, 1997). Avec la montée du chômage, le triptyque «réduction du temps de travail, flexibilité et emploi » a donné matière à une intense activité de réforme en faveur de procédures de négociation plus décentralisées. Le lien entre temps de travail et emploi n'est pas pour autant devenu simple et linéaire : la persistance des débats actuels (autour des 35 heures notamment) laisse deviner la complexité des relations entre ces deux variables. Le temps est en effet une construction sociale dont les dimensions et les fonctions sont multiples. Il est tout à la fois un élément de mesure du travail abstrait, objet d'échange, un facteur de limitation et de protection du travail concret et un paramètre organisationnel pour 1'entreprise. Mais il est aussi une composante centrale du monde vécu des individus, puisque le partage entre temps de travail et temps libre définit les conditions de la vie quotidienne et structure les rythmes sociaux (Mercure, 1995 ; Naville, 1972).

À l'heure actuelle, le temps est surtout perçu comme un médium entre le travail et l'emploi. Il est un moyen de définir les conditions de répartition du travail, les rémunérations et les statuts professionnels. Mais il est aussi le support de confrontations multiples entre individus, ou entre groupes sociaux, confrontations dont l'enjeu est la place respective à accorder au travail, à la vie personnelle et familiale, aux relations de sociabilité et, plus encore, aux types de concordance entre ces différents temps (Supiot, 1995 ; Thoemmes, de Terssac, 1997b).

La question est alors, autant de savoir à quelles conditions (sociales, juridiques, économiques...) le temps de travail peut s'imposer comme un élément déterminant de la politique de 1'emploi que de définir la place de l'emploi dans les négociations concrètes en entreprise.

Pour répondre à ces questions, nous examinerons la façon dont l'emploi s'est immiscé dans la négociation sur le temps de travail ainsi que le rôle de cette variable dans la construction et l'économie des accords. Notre objectif est double. Il s'agit d'une part de chercher à

\footnotetext{
* M.L. Morin, est chercheur CNRS au LIRHE, G de Terssac est directeur de recherche au CNRS, CERTOPCNRS et Univ. Toulouse Le Mirail, J. Thoemmes est ATER à l'Université Toulouse Le Mirail et membre du CERTOP:
} 
comprendre la nature des règles temporelles négociées, compte tenu des divers dispositifs juridiques utilisables. Nous souhaitons, d'autre part, mettre à jour la fonction effective de l'emploi dans la régulation de la négociation. Notre hypothèse est que le temps de travail est de plus en plus un objet de marchandage qui sert à obtenir autre chose en rapport avec l'emploi. Ce dernier a, en bref, statut de valeur d'arbitrage qui favorise la production d'arrangements temporels variés.

Sur 1e plan méthodologique, plusieurs niveaux d'analyse ont été croisés. Notre regard est d'abord juridique et statistique. Dans la première partie de cet article, nous considérons la façon dont l'emploi a été inscrit dans les dispositifs légaux ainsi que dans les accords sur le temps de travail. L'étude systématique de la genèse des réformes juridiques et de leur contenu (Morin, 1998) est, à cet égard riche d'enseignements. L'étude des accords conclus dans une région après la loi quinquennale du 20 décembre 1993 nous permettra par ailleurs de préciser comment les dispositifs sont négociés (Thoemmes, de Terssac, 1997a Morin et alii, 1996). S'il croise encore droit et sociologie, le second niveau d'analyse se veut plus attentif encore aux pratiques mises en œuvre pour mêler les deux objets qui nous occupent. La seconde partie s'instruit ainsi des enseignements de monographies d'entreprises ayant négocié, au nom de l'emploi, sur le temps de travail. Nous pourrons alors faire retour sur les textes juridiques eux-mêmes pour alimenter une nouvelle réflexion sur les modes d'usage de l'emploi dans la construction des arrangements temporels.

\section{I- Du travail à l'emploi : l'enjeu des accords sur le temps de travail.}

Les juristes mettent souvent l'accent sur l'intégration de la dimension «emploi » dans le droit du travail contemporain (Pélissier et alii, 1997). À un droit centré sur la protection du travail comme activité humaine concrète et sur la définition de son statut se substitue aujourd'hui un droit plus attentif aux conditions de maintien ou de création d'emplois. Le droit participe ainsi à la réalisation de l'objectif constitutionnel en vertu duquel chacun a le droit d'obtenir un emploi »(Benoit, 1995). Une telle inflexion est particulièrement nette dans le domaine du temps de travail ${ }^{1}$. Jusqu'au milieu des années soixante-dix, les questions de réduction et d'aménagement de ce temps étaient traitées au chapitre consacré à l'amélioration des conditions de travail. Favorisée par une politique de partage des gains de productivité, la réduction tendancielle de la durée du travail était alors un gage de progrès social. Depuis 1982, et sous 1'impact de la crise économique, les termes du débat ne sont plus du tout les mêmes.

\section{1-1 Les réformes successives du régime du temps de travail depuis 1982.}

Les réformes successives opérées depuis 1982 ont conduit à une véritable déconstruction du mode de régulation du temps de travail en vigueur au cours de la période de croissance d'après-guerre (Gavini, Toller, 1994). Régi par des normes tutélaires qui encadrent le pouvoir de direction du chef d'entreprise (Jeammaud, 1982), le temps de travail est devenu

\footnotetext{
${ }^{1}$ Cf. la loi du 27 décembre 1973 sur les horaires variables, la loi du 6 juillet 1996 sur le repos compensateur
} ou encore l'accord interprofessionnel de 1975 sur I 'amélioration des conditions de travail. 
un objet de négociation obligatoire au sein des entreprises. La reconnaissance de négociations dérogatoires a permis par ailleurs à ces dernières d'adopter des cadres temporels hétérogènes et flexibles et de rompre par là même avec une régulation centralisée et uniforme. La définition de l'horaire collectif se conjugue ainsi aujourd'hui avec de multiples possibilités d'individualisation du temps de travail. Le développement du temps partiel en est certainement la manifestation la plus tangible.

Trois étapes au moins se sont succédées, qui éclairent la façon dont l'emploi s'est inscrit dans les textes juridiques.

La première est marquée par l'ordonnance du 16 janvier 1982. Dans le cadre d'une politique affichée de réduction du temps de travail au service de l'emploi (objectif de réduction à 35 heures avec réorganisation du travail), elle reprend pour partie les termes de l'accord du 13 juillet 1981. Celui-ci autorise l'échange d'une réduction de la durée légale contre la possibilité d'un aménagement négocié du temps de travail et ceci afin de procurer plus de flexibilité aux entreprises. En accordant ainsi les premières possibilités de dérogation, cette ordonnance change le sens et la portée de la dynamique contractuelle : celle-ci n'est plus nécessairement synonyme de négociation d'avantages supplémentaires. La mise en œuvre de l'ordonnance s'est traduite cependant par une dissociation des termes de 1'échange. Pour les organisations syndicales, l'obtention immédiate des 39 heures sans perte de salaire représentait d'abord, et de façon traditionnelle, un avantage social conquis à la faveur d'une situation politique plutôt favorable. Dans les négociations, réduction et aménagement du temps de travail n'ont pas toujours été liés. L'emploi, qui a servi de toile de fond à la réforme, n'a pas été le premier moteur de sa réalisation, ni un résultat tangible. En d'autres termes, s'il a servi de référence pour conduire les changements de 1981 et 1982, l'emploi n'a pas été de fait le premier moteur des négociations.

La seconde étape consacre la logique de la flexibilité aux dépens de la réduction du temps ouvré. À la suite de l'échec de la négociation de décembre 1984, l'objectif de baisse générale de la durée du travail est abandonné au profit d'actions incitatives. La réduction du temps de travail acquiert alors un statut de contrepartie aux nouveaux modes d'aménagement qui permettent de créer des emplois (loi du 26 février 1986). Avec la loi du 11 juin 1987, priorité est donnée plus que jamais à la flexibilité négociée dans l'entreprise. La réduction du temps de travail devient facultative. À côté des négociations routinières sur les horaires, les ponts et les congés ${ }^{2}$, l'organisation du travail, domaine où s'exerce traditionnellement le pouvoir de direction de l'employeur, fait l'objet également de compromis avec les organisations syndicales. Dans ces négociations, les acteurs sont convoqués pour définir et harmoniser des rythmes de production, adopter des cadres temporels multiples et différenciés (Ray 1987). Ici, 1'objectif est bel et bien l'efficience économique, qui paraît être, aux yeux de nombreux acteurs de la négociation, le gage du maintien de l'emploi. Autrement dit, le temps de travail devient un outil de gestion des

\footnotetext{
${ }^{2}$ Ces types de compromis négociés restent aujourd'hui les plus fréquents : ils représentent $49 \%$ des accords en 1994 et 1995 contre, il est vrai, en 1992 et $56 \%$ en 1993.
} 
entreprises dont l'usage doit favoriser l'adaptation aux contraintes du marché (Barthélémy, 1990). Mais, faute d'accord sur la compensation salariale et le partage des gains de productivité, la politique de réduction du temps de travail reste au point mort.

La troisième étape se situe au début des années quatre vingt dix. Dans un contexte de marché du travail déprimé, l'articulation entre réduction du temps de travail et emploi est au centre des négociations et des réformes. Certaines entreprises expérimentent des accords de partage du travail offensifs (création d'emplois) ou défensifs (limitation des impacts des plans sociaux) (Bloch London et alii. 1994; Ray, 1994). C'est également le temps de l'incitation au développement du temps partiel de manière à mieux «partager » l'emploi ${ }^{3}$. Dans cette même logique, le chapitre de la loi quinquennale relatif à l'organisation du temps de travail emboîte plusieurs dimensions et traduit, plus exactement, le souci d'intégrer dans la loi la force des liens entre réduction du temps de travail, flexibilité et emploi. Si l'on excepte le cas des contrats de solidarité/réduction du temps de travail, aucun texte n'avait été auparavant conçu en ce sens. Comment cela se décline t-il concrètement? L'incitation entre les termes du triptyque précédent apparait d'abord dans les dispositifs d'annualisation. Elle est plus nette encore dans les dispositifs qui permettent aux entreprises de bénéficier d'une aide de l'État à condition de s'engager à maintenir ou créer des emplois. Ces instruments sont, soit des dispositifs-individuels de partage du travail (incitation au développement du temps partiel et des préretraites) (Favennec-Héry, 1994), soit des dispositifs collectifs pour lesquels l'aide de l'État est destinée à faciliter la compensation salariale (article 39 de la loi quinquennale élargi par la loi Robien de $1996^{4}$. On notera que la loi Robien est le seul texte - exception faite, là encore, des anciens contrats solidaritéréduction du temps de travail - qui subordonne l'aide de l'État à la conclusion d'un accord collectif comportant des engagements relatifs à l'emploi. Mais, conséquence toujours des inflexions imputables à la loi quinquennale, se généralise la possibilité d'annualiser le temps de travail et de le gérer collectivement ou individuellement selon des modalités variables. Le calcul et la répartition du temps ouvré dans un cadre hebdomadaire ne sont donc plus qu'une option parmi d'autres. La loi quinquennale promeut plus explicitement encore de multiples formes d'individualisation du temps comme le temps partiel annualisé (Bloch-London et alii, 1996), le repos compensateur de remplacement, le compte épargne temps ou encore certains types inédits de modulation des horaires.

Produit de réformes successives et ponctuelles, la normalisation juridique du temps de travail aboutit aujourd'hui à une "sur-réglementation » d'une extrême complexité dont l'objectif est en fait la dérégulation (Elbaum, 1996). Conséquence : le temps de travail est maintenant en miettes (Freyssinet, 1997). C'est dans ce cadre évolutif que 1'emploi est

\footnotetext{
${ }^{3}$ Cf. la loi du 3 janvier 1991 ainsi que 1a loi du 31 décembre 1992 sur les aides à la création ou à la transformation d'emploi à temps partiel et sur les préretraites progressives.

${ }^{4}$ L'article 39 prévoyait un accord d'annualisation /réduction du temps de travail avec réduction de salaire. La loi Robien subordonne l'aide de l'Etat à un accord de RDT d'au moins $10 \%$ avec aménagement du temps de travail et un engagement d'embauche correspondant ou de maintien de l'emploi si l'accord est passé dans le cadre d'un licenciement pour motif économique. Il n'y a plus de conditions sur la compensation salariale.
} 
devenu un enjeu central de la négociation du temps de travail. Expression symptomatique d'un tel mouvement, l'accord interprofessionnel sur l'emploi du 31 octobre 1995 est bâti sur une logique d'échange entre réduction de la durée du travail et flexibilité. Bien qu'il n'innove guère sur le fond (Favennec Héry, 1997) et qu'il n'ait été guère suivi d'effets au niveau des branches, le compromis de 1995 consacre plus que jamais l'emploi comme objectif central de la régulation du temps de travail.

\section{1-2 La place de l'emploi dans les accords}

Les divers dispositifs réglementaires dont il vient d'être question, ont été les supports de négociations souvent complexes au sein desquelles l'emploi occupe une place nouvelle. Ces dispositifs ont en commun trois caractéristiques : une représentation du temps de travail qui s'associe à celle de l'emploi, une moindre importance accordée à la réduction de la durée du travail et, enfin, une individualisation des normes temporelles. Comment cela se traduit-il concrètement dans les pratiques de négociation?

On observe au niveau national, que les accords ayant un objectif explicite de maintien ou de création d'emploi, tels qu'ils sont recensés dans le bilan de la négociation collective ${ }^{5}$, sont en constante augmentation. Ces arrangements s'inscrivent sans conteste dans les orientations de la loi quinquennale. En effet, il s'agit soit d'accords de réduction collective du temps de travail liés à des mesures de flexibilité destinées à améliorer la compétitivité des entreprises, soit d'accords de réduction et annualisation individuelles du temps de travail (passage à temps partiel, préretraite). Pour affiner ce type de constat global, nous avons analysé les accords qui ont pu remonter, sur moyenne période (1984-95), jusqu'à une Direction régionale du travail et de l'emploi (Thoemmes, de Terssac, 1997a) ${ }^{67}$

La première classe (29 accords) intègre le travail posté d'un côté, les repos compensateurs et les heures supplémentaires de 1'autre. Ces trois thèmes sont fortement corrélés. Cette classe pourrait renvoyer à une logique de négociation du travail posté visant à compenser les impacts liés à l'extension de ce type d'organisation du travail. La durée d'utilisation des équipements est en effet traitée conjointèrent avec la gestion des heures supplémentaires et du repos compensateur. Nous proposons de nommer «temps de l'extension compensée » le type d'objet au cour des transactions ici repérées. La seconde classe (31 accords) rassemble l'emploi, le temps partiel, la modulation, la réduction de la durée du travail et les

\footnotetext{
${ }^{5}$ Selon les bilans de la négociation collective du Ministère du travail, on compte 892 accords en 1996 contre 693 en 1995, 576 en 1994 et 387 en 1993. Plus généralement, en 1995, le temps de travail fait l'objet de $42 \%$ des accords d'entreprise L'aménagement du temps dc travail est traité dans $58 \%$ de ces accords en 1995. Ce pourcentage est en constante augmentation au cours de ces dernières années. Les dispositifs d'adaptation aux fluctuations d'activité sont traités, en 1995 toujours, dans $38 \%$ des accords et la modulation ou l'annualisation est présente dans un accord sur cinq. Sont exclus ici du décompte les accords qui peuvent avoir un effet sur l'emploi en l'absence de clauses explicites.

${ }^{6}$ Nous nous tiendrons ici à la présentation des résultats issus du traitement de 140 accords conclus, après la loi quinquennale, en 1994 et 1995 donnant lieu à 4 classes d'accords.

${ }^{7}$ L'analyse multivariée permet de mettre en évidence quatre classes d'accords qui constituent autant de pôles de négociation ou, plus exactement, de logiques de marchandage (tableau 1).
} 
horaires individualisés. Est enjeu ici «le temps de l'emploi flexible ». La troisième classe (59 accords) va réunir essentiellement les reconductions simples de dispositifs antérieurs et renvoie à une logique du « temps confirmé ». Cette classe ne présente aucune association significative entre temps, emploi et salaire. Enfin, la quatrième classe ( 21 accords) traite des périodes d'activités non professionnelles : c'est le «temps des congés ». Cette dernière classe est par ailleurs significativement corrélée aux questions de salaire.

Tableau 1. Les 4 pôles de négociation sur le temps de travail (classe 1 à 4: pour 140 accords), en fonction de la structure externe (articulation temps, salaire, emploi) et de la structure interne (28 thèmes) du temps de travail (méthode: analyse multi-variée).

\begin{tabular}{|c|c|c|c|c|c|}
\hline $\begin{array}{l}4 \text { classes sur } 140 \\
\text { accords }\end{array}$ & \begin{tabular}{ll}
\multicolumn{2}{|l}{ structure externe: } \\
(temps, $\quad$ salaire, \\
emploi)
\end{tabular} & $\begin{array}{l}\text { structure } \\
\text { interne: thème } 1\end{array}$ & thème 2 & thème 3 & thème 4 \\
\hline I: 29 accords & $\begin{array}{l}\text { temps de travail seul } \\
+\end{array}$ & $\begin{array}{l}\text { repos comp. } \\
+++\end{array}$ & $\begin{array}{l}\text { heures supp. } \\
+++\end{array}$ & $\begin{array}{l}\text { travail en } \\
\text { équipes } \\
+++\end{array}$ & $\begin{array}{l}\text { travail de } \\
\text { nuit } \\
+++\end{array}$ \\
\hline II : 31 accords & $\begin{array}{l}\text { temps et emploi } \\
+++\end{array}$ & $\begin{array}{l}\text { temps partiel } \\
+++\end{array}$ & $\begin{array}{l}\text { modulation } \\
+++\end{array}$ & $\begin{array}{l}\text { horaires } \\
\text { variables } \\
+++\end{array}$ & $\begin{array}{l}\text { réduction } \\
\text { durée } \\
+++\end{array}$ \\
\hline III:59 accords & $\begin{array}{l}\text { sans lien } \\
0\end{array}$ & $\begin{array}{l}\text { recond. durée } \\
+++\end{array}$ & $\begin{array}{l}\text { recond. orga. } \\
+++\end{array}$ & $\begin{array}{l}\text { fin de semaine } \\
++\end{array}$ & $\begin{array}{l}\text { dates } \\
\text { congés } \\
+\end{array}$ \\
\hline IV:21 accords & $\begin{array}{l}\text { temps et salaire } \\
+\end{array}$ & $\begin{array}{l}\text { congés part. } \\
+++\end{array}$ & $\begin{array}{l}\text { récup. Ponts } \\
+++\end{array}$ & $\begin{array}{l}\text { dates ponts } \\
+++\end{array}$ & $\begin{array}{l}\text { durée } \\
\text { congés } \\
+++\end{array}$ \\
\hline
\end{tabular}

\begin{tabular}{llll}
\hline légende & corrélation & valeur test & risque \\
\hline-- & négative & khi2 $>\mathbf{1 0 , 8}$ & $1 / 1000$ \\
-- & négative & khi2 $>6,6$ & $1 \%$ \\
- & négative & khi2 $>3,8$ & $5 \%$ \\
0 & indépendance & khi2 $=0$ & \\
+ & positive & khi2 $>3,8$ & $5 \%$ \\
++ & positive & khi2 $>6,8$ & $1 \%$ \\
+++ & positive & khi2 $>10,8$ & $1 / 1000$ \\
\hline
\end{tabular}


Comment interpréter ces résultats du point de vue du rôle de l'emploi, tant dans son inscription dans les textes juridiques, que dans la place qu'il occupe dans les accords? Trois pistes interprétatives peuvent être ouvertes.

Premièrement, il semble que l'emploi apparaisse comme une monnaie d'échange dans les négociations sur le temps de travail : le temps de travail est un objet de marchandage au service de l'emploi. Les termes de l'échange pourraient s'énoncer ainsi: l'employeur accepte de traiter des questions d'emploi, sous réserve que les salariés acceptent une certaine flexibilité (modulation et temps partiel); réciproquement, les salariés acceptent la remise en cause des repères temporels habituels et le temps partiel (imposé ou choisi), à condition que l'employeur prenne en compte la situation d'emploi. L'emploi servirait ici à se mettre en négociation sur le temps de travail, voire à produire des compromis acceptables par les parties en présence. Pour autant, cela signifie-t-il que les parties en présence sont d'accord sur une même vision du temps de travail?

Deuxièmement, la multiplicité des possibilités d'aménagement du temps de travail, en réponse aux exigences de flexibilité, se traduit par l'éclatement du temps de travail : ceci pose pour l'individu un problème d'articulation entre une pluralité de temporalités. L'extension de la disponibilité du salarié à l égard de l'entreprise, ne se traduit-elle pas par une perte de maîtrise de la gestion de sa disponibilité personnelle? La détermination des conditions de la vie quotidienne et du travail concret demeure donc un enjeu central ${ }^{8}$.

Troisièmement, il apparaît que, en l'absence d'incitation d'origine réglementaire ou de décision législative, la réduction collective de la durée du travail demeure une pratique exceptionnelle : $5 \%$ seulement des accords conclus en 1994 et 1995 débouchent sur un tel cas de ligure. Preuve, s'il en fallait encore une, de la prégnance de l'enjeu gestionnaire dans les négociations sur le temps de travail et de la difficulté de la question de la compensation salariale.

\section{II- L'emploi : une valeur d'arbitrage dans la négociation du temps de travail}

Placer la négociation du temps de travail sous l'égide de l'emploi, c'est en effet lui conférer une valeur d'arbitrage entre les autres enjeux, ceux de la production, ceux du partage des gains de productivité, ceux des conditions du travail concret, dans la vie quotidienne et sociale. Ce rôle dépend en réalité de la façon dont cette valeur est mobilisée tant dans la négociation elle-même que dans le texte des accords. C'est pourquoi nous analyserons tout d'abord, au travers de trois situations types, la manière dont l'emploi est utilisé en pratique dans les négociations sur le temps de travail, comme « catalyseur », comme « régulateur », comme «écran ». Nous analyserons ensuite et de façon plus générale, de quelle manière il est mobilisé dans les textes des accords, comme simple «justification », comme le résultat d'un «plan de gestion finalisée », enfin comme matière à « engagement » (Lyon Caen, 1996).

\footnotetext{
${ }^{8}$ Sur ce point il faudra suivre les conditions de mise en oeuvre du compte épargne de temps et les projets de réforme du temps partiel.
} 


\section{2-1 Les chemins de la négociation}

Dans la négociation sur le temps de travail, l'emploi intervient de manière différente: c'est ce que révèle l'analyse monographique portant sur une dizaine d'entreprises ${ }^{9}$. De cette base de données, nous extrayons trois cas de figure, qui illustrent le rôle différentiel de l'emploi dans les négociations sur le temps de travail. Tout d'abord, il peut déclencher une négociation sur le temps de travail, sans pour autant faire l'objet de la négociation; il a alors un rôle de «catalyseur». L'emploi peut ensuite être au coeur d'une négociation sur le temps de travail: il constitue le préalable à la négociation et la condition d'un accord sur le temps de travail; il a un rôle de «régulateur » de la négociation. Enfin, l'emploi, tout en étant au coeur de la négociation, peut être une occasion d'opposition entre les parties en présence et compromettre l'élaboration d'un accord sur le temps de travail: il a alors un rôle « d'écran ».

\section{A- L'emploi « catalyseur » de la négociation sur le temps de travail}

Dans une entreprise du secteur pharmaceutique, la négociation porte sur le repos compensateur: il s'agit d'un accord banal, obtenu dans le cadre d'un recours à l'une des dispositions de la loi quinquennale; cet accord, signé en 1995 par la direction et l'ensemble des organisations syndicales présentes (CFTC et CGT qui est majoritaire), vise à légaliser un arrangement temporel tacite antérieur. Le résultat de cet accord est moins banal, si l'on considère les chemins qu'emprunte la négociation: ils révèlent le rôle de l'emploi comme « catalyseur » de la négociation sur les heures supplémentaires.

- Tout d'abord le recours aux heures supplémentaires avait fait l'objet d'un arrangement temporel tacite entre les salariés et l'employeur: le dépassement du volume d'heures supplémentaires légal était contourné par une transformation de ces heures en repos regroupé sous formes de congés supplémentaires; ces congés pouvaient atteindre 12 semaines dans l'année.

- Ensuite, le contexte de l'emploi conduit les organisations syndicales à remettre en cause cet arrangement pour faciliter l'emploi. En effet, une restructuration de l'entreprise en 1993 entraîne une vague de licenciements (100 salariés sur 270); de plus, la réduction programmée des activités (de 5\% par an) fait craindre aux salariés une nouvelle vague de licenciements. D'où un recours à l'inspection du travail à la demande de l'une des organisations syndicales; le raisonnement est le suivant: « si l'inspecteur du travail constate que l'on fait trop d'heures supplémentaires, il n'y a qu'à embaucher ». L'inspection du

\footnotetext{
${ }^{9}$ Ces monographies ont été réalisées au travers en 1996 et en 1997 d'entretiens avec les responsables de la direction et les différentes organisations syndicales: en moyenne 3 entretiens enregistrés ont été réalisés par entreprise, de 2 heures environ.
} 
travail intervient et sanctionne le dépassement des heures supplémentaires. La réponse de la direction n'est pas de favoriser l'embauche mais "puisque l'inspecteur du travail intervient, on devient légal (...), et on supprime le repos compensateur non légalisé». L'employeur décide alors de remettre en cause les termes de l'arrangement antérieur.

- Enfin, l'accord d'entreprise obtenu se traduit dans une légalisation de l'arrangement tacite: d'une part il codifie et mesure le recours aux heures supplémentaires qu'il inscrit dans le cadre du repos compensateur de remplacement. D'autre part, il définit de nouvelles règles de recours aux heures supplémentaires en évitant qu'elles soient comptabilisées dans le contingent légal; il instaure ainsi un nouveau mode de régulation des heures supplémentaires qui par son caractère légal devient incontestable.

La signification de cet accord n'est pas évidente: quel rôle l'emploi a-t-il joué? S'agit-il d'un retour à la case départ, comme l'indique l'employeur: "notre accord, vise à rétablir une pratique antérieure qu'une situation exceptionnelle avait déstabilisée »? Cette interprétation n'est pas tout à fait exacte pour deux raisons: tout d'abord cet accord inscrit le recours aux heures supplémentaires dans un cadre légal et institutionnalise une certaine forme de flexibilité ; cela n'est pas sans importance si l'on considère que la séquence de travail conduit à travailler de $10 \mathrm{~h}$ à $19 \mathrm{~h}$, alors que les plages horaires officielles sont dans ce secteur de $8 \mathrm{~h}$ à $17 \mathrm{~h}$; ensuite, l'accord se traduit par un non traitement de la question de l'emploi qui a pourtant été à l'origine de la négociation : l'emploi a donc joué un rôle de catalyseur qui a déclenché la négociation, sans se retrouver dans le contenu de l'accord. Cette interprétation du rôle de l'emploi s'appuie sur le changement des termes de la discussion: non plus la création d'emplois contre la suppression d'heures supplémentaires, mais le maintient des heures supplémentaires (avec un coût probablement réduit) contre du repos compensateur ; dès lors, l'emploi se trouve relégué en dehors du domaine de la négociation en cours.

\section{B- L'emploi comme « instrument de régulation » de la négociation sur le temps de travail?}

Dans cette entreprise de la mécanique d'environ 1300 salariés, l'accord conclu en 1996 se caractérise par un échange entre réduction de la durée du travail et maintien de l'emploi d'un côté, et aménagement des horaires, auquel s'ajoute une faible perte de salaire, de l'autre; cet accord a été signé par l'employeur, la CFDT et FO, mais non par la CGT. L'importance de cet accord ne réside pas seulement dans son contenu, mais aussi dans l'histoire de sa construction et dans les termes de l'échange qui ont été modifiés par cette histoire.

Premièrement une composante essentielle du contenu de l'accord concerne un échange désormais classique entre la réduction de la durée du travail, contre l'aménagement des horaires. D'un côté la durée du travail hebdomadaire diminue de 2 heures environ pour les équipes alternantes (de matin et d'après midi) et de presque 6 heures pour l'équipe de nuit fixe (la durée de chacune des 5 nuits consécutives diminue de plus de 1h15mn). De l'autre, 
on assiste à une extension de la délocalisation des périodes de travail du fait de l'augmentation de la durée d'utilisation des équipements : on commence une heure plus tôt le matin et on finit une heure plus tard le soir; de plus on assiste à l'apparition de la modulation des horaires sur l'année (pour faire face aux fluctuation de la demande) « qui peut arriver à tout moment ».

Deuxièmement, cet accord a mis 15 ans pour aboutir, si l'on en juge par la double acceptation du travail du samedi régulier (réalisé par 2 équipes successives, de $5 \mathrm{~h}$ à 20h15) et de la perte de salaire (d'environ 3\%), accompagnant la diminution de la durée hebdomadaire du travail. Ces deux points avaient en effet fait l'objet de nombreuses discussions depuis 1982, qui n'ont jamais débouché sur un accord. Deux tentatives de réduction du temps de travail avec diminution de salaire ont échouées, tant en 1982 qu'en 1984 et les négociations de 1989 et de 1995 concernant le travail du samedi n'ont pas abouti non plus.

Troisièmement, l'emploi occupe une place centrale dans cet accord. Le préambule précise en effet d'une part que cet accord a pour but «de supprimer le plan social engagé et entraînant le licenciement pour motif économique » (de plus de 10\% des salariés) et d'autre part «d'augmenter le temps libre et d'améliorer l'emploi ».

Il n'est pas aisé là non plus de donner une interprétation univoque de cet accord, car la relation entre le temps de travail et l'emploi mérite d'être explicitée. Deux interprétations de cette relation sont possibles: la première pourrait permettre de conclure que le temps de travail est un instrument de régulation de l'emploi, qu'il sert à maintenir. C'est ce qui semble se dégager pour deux raisons qui se combinent: tout d'abord, il y a bien acceptation par les signataires d'une détérioration des repères temporels antérieurs (travail du samedi, travail plus tôt le matin et plus tard le soir) contre le maintien de l'emploi ; ensuite, il y a bien acceptation de l'augmentation de la flexibilité (modulation sur l'année des périodes de travail) et de la perte de salaire contre la réduction de la durée hebdomadaire.

Mais une deuxième interprétation pourrait nous amener à considérer que l'emploi est un instrument de la régulation de la négociation sur le temps de travail. Cette interprétation se nourrit des faits suivants : rappelons, qu'aucun accord n'avait été obtenu au cours des 15 dernières années sur le temps de travail et qu'il faut attendre 1996 pour que les partenaires sociaux trouvent un accord sur le travail du samedi; la négociation n'a abouti que lorsque la question de l'emploi intervient dans la discussion. En effet, alors qu'en 1995 la négociation avait repris avec deux des organisations syndicales (CFDT et FO) qui s'apprêtaient à accepter le travail du samedi, la troisième organisation (la CGT) a décidé de consulter les salariés et à l'issue d'un référendum dans lequel 2/3 des salariés s'expriment contre un mandat de «négociation du travail de samedi après-midi »; désavouées, les deux autres organisations ont quitté la table des négociation et la direction estimant que l'objectif majeur de la négociation n'a pu être atteint a constaté l'échec de la négociation (constat de désaccord). 
Un événement semble avoir débloqué la situation: l'apparition début 1996 d'un plan social prévoyant la réduction des effectifs qui a entraîné, semble-t-il, la reprise des négociations et finalement la signature de l'accord : «Dans le projet de plan social, nous représentons comme solution pour éviter les licenciements de négocier à nouveau un accord d'aménagement et réduction du temps de travail, (..), mais c'est la dernière chance », (représentant de la direction).

Entre temps, les termes de l'échange ont effectivement changé: au triptyque des négociations antérieures basées sur les relations durée du travail, flexibilité, salaire s'ajoute l'emploi; celui-ci joue un rôle de régulateur: «Au niveau des licenciements, si on peut en sauver quand même, il faut faire l'effort », (entretien salarié non-syndiqué). L'emploi ne concerne pas directement la nature de l'arrangement temporel trouvé, mais bien la condition de son invention: c'est la raison pour laquelle nous pourrions interpréter, dans ce cas précis, le rôle de l'emploi comme un instrument de régulation de la négociation sur le temps de travail.

\section{C- L'emploi comme écran: une négociation du temps de travail bloqué}

Dans ce regroupement régional d'établissements du secteur de la santé, plusieurs milliers de salariés travaillent en continu, pour la plupart des femmes: un accord national stipule en 1991 la réduction de la durée du travail de nuit de 39 heures à 35 heures. Ce même accord signé par la CFDT propose simultanément la création, au niveau national, de 4000 emplois, afin de compenser les heures perdues par la réduction de la durée du travail de nuit.

On pourrait penser à une application sans difficulté de cet accord au niveau régional, puisqu'il propose non seulement de réduire la pénibilité du travail de nuit, mais en plus, il constitue un engagement au regard de la création d'emplois. Pourtant, au niveau régional l'application s'avère difficile. En effet, l'accord national préconise que la création d'emplois est conditionnée par une réorganisation de la journée de travail, afin d'augmenter la disponibilité et la productivité du personnel: «La réduction de la durée du travail peut être compensée partiellement par une meilleure adéquation des moyens en personnel à l'activité réelle des services » (avenant à l'accord de 1991). Les horaires de jour sont donc censés devenir plus souple en vue d'accroître la disponibilité des salariés: «Donc, il y aura une journée où l'agent pourrait faire 6 heures de travail au lieu de 8 heures, (...), et le surlendemain il pourra faire 10 heures», (entretien avec un représentant de la direction). Un bilan national effectué en 1994 sur la réduction du travail de nuit à 35 heures, montre qu'une partie importante des établissements au niveau local n'a toujours pas appliqué l'accord national (environ un sur trois). Dans notre cas, l'application prévue entraînait la création de plusieurs centaines d'emplois: cette revendication formulée par les organisations syndicales s'est heurtée à un refus de la direction; plusieurs tentatives de négociation ont échoué et avaient provoqué notamment en 1994 des manifestations importantes. 
Une première raison évidente explique l'échec des négociations sur la réduction du travail de nuit: la direction ne veut pas s'engager formellement à conforter l'emploi; plus exactement, les mesures touchant l'emploi ne peuvent être considérées pour elle comme les seules justifications des actions concernant le temps de travail. Les salariés, quant à eux, font de l'engagement sur l'emploi le résultat attendu des mesures touchant le temps de travail.

Une deuxième raison peut expliquer l'échec des négociations: elle a trait aux termes de l'échange. Côté direction, il ne peut y avoir d'engagement précis sur l'emploi qu'en contrepartie d'aménagements du temps de travail, c'est à dire de modifications sur les horaires notamment de jour. Côté salariés, la réduction du travail de nuit doit être accompagnée par des mesures sur l'emploi, mais en aucun cas elle ne doit remettre en question les repères temporels habituels (heures de début et de fin de poste notamment). Trois arguments sont évoqués par les salariés: (a) le refus du contrôle des horaires, (b) le refus de voir ses repères temporels modifier l'articulation entre plusieurs temporalités sociales, enfin (c) le refus devoir abandonner certains avantages, liés aux compensations consécutives au travail de nuit.

Tout d'abord (a) la réduction de la durée du travail de nuit à $35 \mathrm{~h}$ et la réorganisation globale implique une mesure quantifiée de la durée du travail: "Cela suppose un badgage informatique, or la culture de l'établissement n'est pas au pointage » (représentant de la direction). En effet, la mesure exacte des durées du travail et des opérations sur le plan quantitatif rencontre l'hostilité des salariés qui renvoient à l'administration l'argument d'une qualité de travail, difficilement quantifiable: "Voilà ce qui est l'administratif, il quantifie, il compte en chiffre, en argent, en norme, en kilo, en litre », (entretien avec une salariée). Ensuite (b) le refus d'échanger la réduction contre l'aménagement: les horaires de travail obéissent en général à des configurations suivantes. Les plages horaires du personnel de jour sont de $7 \mathrm{~h}$ à $15 \mathrm{~h}$, de $8 \mathrm{~h}$ à $16 \mathrm{~h}$ et de $12 \mathrm{~h}$ à $20 \mathrm{~h}$ ( $80 \%$ des salariés). Le personnel de nuit travaille de $20 \mathrm{~h} 30$ à $7 \mathrm{~h}$ (20\% des salariés). Alors que l'application de l'accord national permettrait raccourcir le travail de nuit de $10 \mathrm{~h} 30$ à une durée de 8 heures, l'aménagement consécutif des horaires de jour pour l'après-midi de $13 \mathrm{~h} 30$ à $21 \mathrm{~h} 30$ est refusé par les salariés. La sortie des salariés à $21 \mathrm{~h} 30$ et l'arrivée au domicile à $22 \mathrm{~h} 30$ ne sont pas acceptées par certains, qui ne souhaitent pas utiliser les transports en commun à cette heure tardive (sécurité). D'autres refusent de commencer plus tôt l'équipe du matin, à cause de l'absence de transport urbain ou à cause d'un lever trop matinal, notamment pour les enfants: «Le personnel de jour ne veut pas qu'on l'embête avec des changements pour arranger le travail de nuit», (entretien salariée). Enfin (c), il n'est pas sûr que les principaux intéressés veuillent raccourcir les heures de nuit. Le service traditionnel de nuit alterne entre des périodes où le nombre de nuits consécutives est élevé (5 nuits), avec des périodes où ce nombre est faible ( 2 nuits). Donc, sur 14 nuits seules 7 nuits au maximum sont travaillées par les individus. La longueur des postes (10h30) implique, en plus, un certain nombre de journées de récupération. Ces récupérations seraient diminuées avec 
l'introduction des 35 heures: «Avec les 35 heures (...), elles sont obligées de rendre des heures, ce qui veut dire une diminution des récupérations, (...). Alors pourquoi imposer des horaires à des gens, alors qu'ils en sont satisfaits », (entretien, salariée). L'usage des périodes de récupération rend donc difficile l'acceptation de la réduction du travail de nuit. Pour comprendre comment l'emploi est concrètement mis en jeu dans la négociation du temps de travail, cette analyse des chemins de la négociation doit être complétée par celle de la portée de l'objectif emploi, lorsqu'il apparait dans les accords.

\section{2-2 La portée de l'objectif emploi et ses limites}

Sur la base des enseignements précédents, tentons maintenant de revenir sur des questionnements plus généraux en nous appuyant à nouveau sur une lecture de textes juridiques récents. Dès lors, on constate également, que 1'ernploi - qui relève en droit du pouvoir de gestion du chef d'entreprise - peut revêtir une valeur d'arbitrage plus ou moins forte selon les enjeux de la négociation du temps de travail. Comme l'illustrent les trois monographies précédentes, i1 n'est pas rare que le sociologue constate que des accords motivés par des objectifs similaires s'accommodent en fait d'une variété de pratiques. Aux yeux du néophyte en droit, il peut être surprenant en revanche d'observer que le statut de l'emploi soit finalement aussi varié au sein du corpus juridique lui-même. Plus précisément, dans le système réglementaire relatif au temps de travail, l'emploi occupe aujourd'hui trois places différentes. Il est d'abord un objectif de l'annualisation/réduction du temps de travail (article 38 de la loi quinquennale). Il est ensuite une compensation possible lors de la mise en place d'une modulation des horaires. Il peut être, enfin, la contrepartie de l'aide de l'État et, à ce titre, donner matière à engagement. En ce cas, l'aide peut être subordonnée à un accord collectif (loi Robien) ou être attribuée directement et indépendamment d'un tel accord (temps partiel, préretraite progressive). Mais, qu'il ait statut d'obligation légale accompagnant l'adoption de mesures sur le temps de travail ou qu'il serve de contrepartie, l'emploi est considéré comme une condition de validité des accords qui ne peut se satisfaire d'allusions vagues, même si des engagements quantifiés ne sont juridiquement nécessaires que dans les dispositifs légaux qui subordonnent l'aide de 1'État à de tels engagements (loi Robien, projet de loi cadre sur les 35 heures). Ainsi que l'ont mis en évidence A. Lyon-Caen (1996) et M, A. Rostohild-Souriac (1997), l'analyse juridique des accords sur le temps de travail débouche sur un diagnostic similaire : celui de la diversité. Dans les accords, l'emploi peut d'abord être simplement mentionné dans le préambule comme un objectif, sinon comme une simple justification aux côtés de la conciliation entre vie professionnelle et vie du salarié et de la flexibilité des entreprises. Ces accords, qui ne sont assortis d'aucun engagement réel, sont les plus nombreux. ${ }^{10}$ L'emploi

\footnotetext{
${ }^{10}$ Actuellement, l'objectif emploi est ainsi cité dans 1/6 des accords sur le temps partiel. Il est d'ailleurs toujours évoqué en même temps que la volonté de faciliter la conciliation entre vie professionnelle et vie privée et/ou les impératifs de flexibilité, Dans les accords d'annualisation, au côté de l'adaptation de l'entreprise aux variations saisonnières d'activité (2/3 des textes), l'objectif prioritairement affiché est aussi celui de la sauvegarde de l'emploi.
} 
joue ici un rôle d'arbitrage faible car, en ce cas, il n'y a pas d'obligation. L'emploi peut, deuxièmement, figurer dans des accords de gestion plus complets qui intègrent le temps de travail parmi d'autres mesures. Moins fréquents, ces arrangements sont surtout le fait de grandes entreprises ${ }^{11}$. La préservation de l'emploi est bien alors ici le résultat attendu des mesures prises et il y a obligation de moyens, mais pas de résultats. Sachant qu'en droit du travail, les parties ont l'obligation de ne rien faire qui soit de nature à compromettre l'exécution loyale de la convention, se pose alors la question suivante : engager une procédure de licenciement, malgré l'existence d'un accord qui pose un objectif de maintien de l'emploi comme contrepartie d'un ensemble de mesures ? A tout le moins, l'employeur ne doit-il pas s'expliquer préalablement à la décision portant atteinte à l'emploi, sur le sens de l'évocation de l'emploi dans l'accord et sur les conditions de son application? En ce cas, cela n'implique-t-il pas d'ouvrir une renégociation, même si l'affirmation d'un objectif emploi ne suffit pas à lui seul à apporter une garantie d'emploi (M.A. Rotschild Souriac, 1997) ?

L'emploi peut être, troisièmement, la matière d'un engagement ferme, contrepartie des mesures prises sur le temps de travail : il y a en ce cas, obligation de moyens et de résultats. Ces engagements peuvent porter sur le maintien de l'emploi ou sur des créations d'emplois, qui peuvent être ou non chiffrés. Leur portée suscite deux types de questions.

La première a trait aux limites que ces engagements créent dans le domaine du pouvoir de gestion de l'employeur. Il est admis qu'un accord puisse limiter le droit de licencier mais sans pour autant le supprimer. ${ }^{12}$ De même, des engagements d'embauche ne sont pas illicites. Mais si la situation de l'entreprise change, l'employeur peut-il arguer de son pouvoir de gestion pour s'exonérer de ses engagements ? Dans une affaire, où l'employeur avait pris l'engagement d'éviter de nouveaux licenciements avec clause de renégociation, la Cour de cassation a admis qu'il pouvait néanmoins engager directement une nouvelle procédure de licenciement, sans renégocier l'accord, dans la mesure où les licenciements étaient inévitables ${ }^{13}$. Le pouvoir de gestion a donc primé sur l'engagement contractuel mais, il est vrai, que la rédaction de la clause était plutôt obscure. Dans deux autres affaires au contraire, la cour de cassation a, cette fois sans équivoque, sanctionné la violation d'un engagement d'emploi que l'employeur avait méconnu. ${ }^{14}$ Cette dernière solution mérite d'être approuvée. Ces engagements portent sur l'avenir, ils ont pour objet de réduire l'incertitude et en droit, l'imprévision, (i.e. l'incertitude), n'est pas une cause d'exonération des engagements, sauf force majeure. Reconnaître la force de ces engagements contractuels, c'est aussi assurer la confiance qui doit présider à leur exécution. Le développement de la négociation sur l'emploi dépend donc, pour une bonne part, de la clarté et de la force qui peut être reconnue à ces engagements.

\footnotetext{
${ }^{11} \mathrm{cf}$., à titre d'exemple, l'accord EDF de novembre 1995.

${ }^{12}$ SOC. 7juillet 1993, Bull. Civ. V nº 197.

${ }^{13}$ - Soc. 13 décembre 1995, Bull. Civ. V nº 345

14 Soc. 1er avril 1997, Droit Social, 1997, p. 646 Obs RAY.
} 
Le second type de questions a trait aux mesures qualitatives qui sont prises en échange de l'engagement quantitatif sur l'emploi. Ces mesures peuvent en effet conduire à une modification sensible des conditions d'emploi et de travail. Tout d'abord, en raison de l'importance même de ces modifications le recours au volontariat est fréquent. Si le temps partiel nécessite de toute façon un accord du salarié, le volontariat est utilisé plus largement ${ }^{15}$. Les engagements d'emplois lorsqu'ils existent sont alors soumis à l'aléa du nombre de volontaires. Ensuite les modifications du régime du temps de travail, qui s'intègrent souvent dans une négociation dérogatoire, ne sont pas nécessairement plus favorables que les règles légales. Ce type de négociation remet en effet en cause l'ordre public social de deux manières : elle s'affranchissent de la loi qui ne joue plus son rôle de plancher protecteur, elles ne portent pas sur des avantages supplémentaires (c'est pourquoi elles sont soumises à un droit d'opposition).

Lorsque ces dérogations ont pour contrepartie l'emploi, deux interrogations supplémentaires, qui ont une origine commune, apparaissent. Prendre des engagements quantitatifs sur l'emploi, en contrepartie de modifications qualitatives des conditions de travail et/ou de rémunérations des salariés en place, c'est faire de l'emploi un bien public, ou en tout cas un bien collectif auquel doivent concourir les salariés ${ }^{16}$.

D'une part la modification dérogatoire des conditions de travail doit-elle dans ces conditions s'imposer aux salariés, sans qu'ils puissent opposer les conditions de leur contrat individuel, si elles sont plus favorables? La question se pose surtout pour les accords de « réduction de la durée du travail » qui prévoient une diminution de salaire (Bélier 1996). Le recours au volontariat permet d'éviter la question. Mais elle se pose dans les autres cas. Les conditions de la négociation elles-mêmes sont de ce fait déterminantes pour assurer l'acceptation de l'accord par les salariés ${ }^{17}$.

D'autre part, un accord qui comporte un engagement d'emploi en contrepartie de nouvelles contraintes ou de changement des conditions de travail peut-il être considéré comme globalement plus favorable que la loi ou qu'un accord antérieur, quelque soit la remise en cause des avantages préexistants? Dans une affaire qui ne concerne pas le temps de travail la Cour de cassation vient de le décider. ${ }^{18}$ A la protection des salariés fondée sur la mesure de leurs droits et avantages, c'est à dire sur les mesures qualitatives qui sont prises, cette

15 Ex: l'accord du 31 janvier 1997

16 - Cf. l'exemple de l'accord du Crédit Lyonnais du 12 décembre 1996 qui prévoit une contribution de tous les salariés pour compenser le salaire de ceux dont la durée du travail est réduite

${ }^{17}$ - Cf. la circulaire d'application de la loi Robien du 9 octobre 1996 (L.S. A.2 du 18 octobre 1996) qui invite les services de l'Etat a contrôlé ce point. On n'ouvrira pas ici le débat sur la représentativité des signataires et les conditions de signature de l'accord bien qu'il soit évidemment essentiel.

${ }^{18}$ - Soc 27 février 1997, Dr. Social, avril 1997 p. note G. Couturier pour un accord diminuant une prime en contrepartie d'un engagement de maintien de l'emploi. 
décision substitue la protection quantitative de l'emploi, nouvelle aune d'appréciation de l'accord. La consécration d'une telle solution dans le domaine du temps de travail, ferait de l'emploi la valeur d'arbitrage centrale de la négociation du temps de travail, mais ceci ne peut se concevoir que si une portée juridique ferme est donnée aux objectifs d'emploi affichés.

\section{CONCLUSION}

Le passage d'un mode tutélaire de réglementation du temps de travail à un mode négocié largement décentralisé et dérogatoire aux règles légales a permis le développement de la négociation du temps de travail. Les inflexions de la loi quinquennale ont accentué le passage d'un mode de réglementation collectif et homogène du temps de travail à un mode hétérogène et individualisé, qui combinent des cadres différents d'organisation du temps. Dans ce mouvement, la rationalité dominante des textes juridiques, comme des accords sur le temps de travail, est demeurée la flexibilité du temps de travail. La réduction du temps de travail est restée subordonnée à cette rationalité dominante sans être traitée en tant que telle.Cependant l'emploi devient dans les textes juridiques, comme dans les accords, une composante de la négociation du temps de travail qui constitue en quelque sorte une valeur d'arbitrage. Néanmoins, cette valeur d'arbitrage se décline de différentes manières, tant dans les textes juridiques que dans les pratiques négociatoires. Selon les contextes réels, l'emploi peut n'être (a) qu'un élément de la discussion et ne pas être traité en tant que tel: l'emploi a un rôle d'arbitrage faible, même s'il joue un rôle déterminant dans l'entrée en négociation sur le temps de travail; il a alors un rôle de «catalyseur ». L'emploi peut aussi (b) faire l'objet d'engagements fermes en contrepartie de mesures touchant la flexibilité: les négociations évoquées sur le travail du samedi l'illustrent; l'emploi joue ici un rôle de « régulateur » de la négociation sur le temps de travail et constitue la condition du résultat: son rôle d'arbitrage est fort. Enfin (c), l'emploi peut être un objectif qui fait l'objet d'un pronostic au regard des mesures prises sur le temps de travail, sans faire l'objet d'un engagement. C'est ce que nous avons illustré au travers de la négociation visant à réduire le travail de nuit en échange de l'aménagement des horaires et d'une amélioration de la flexibilité: Faute de préciser le sens de l'évocation de l'emploi, il peut être un «écran » de la négociation et s'articuler avec d'autres enjeux liés notamment à la « concordance » des différentes temporalités auxquelles sont soumis les individus. L'un des enjeux des négociations sur les 35 heures sera celui de savoir quel sera le rôle réel de l'emploi. 


\section{Bibliographie}

Barthélémy J. (1990) : «La négociation, outil de gestion de l'entreprise », Droit Social p. 580

Bélier G. (1996) : «Quelques questions autour de la loi Robien » Droit Social p. 1008

Bloch London C., Cahuzac E., Morin M.L., Sublet S., (1996) : "Les accords d'entreprises sur le temps partiel de 1992 à 1995 trois logiques d'utilisation », Paris, Ministère du Travail, Premières Synthèses n96-11, Dares

Bloch London C., Boisard P., Boulin J.Y., Coutrot T., (1994) : «Les expériences d'ajustement des salaires et de partage du travail », Paris, Ministère du Travail, Premières synthèse $11^{\circ} 94 / 43$, Dares.

Elbaum M. (1996) : « La réduction du temps de travail un avenir à quelle conditions ? », Esprit, $\mathrm{n}^{\circ} 226$ p. 64

Favennec Héry F. (1994), Le travail à temps partiel », Droit social, no 2, pp. 165-175.

Favennec Héry F. (1996), L'accord interprofessionnel du 31 Octobre 1995 sur 1'emploi,

Droit social, 12, pp. 999-1007.

Freyssinet J. (1997) : Le temps de travail en miette, Editions de l'Atelier

Gavini C., Tollet, J.Y., (1994) : «La durée du travail, construction et déconstruction d'une norme » Droit Social p. 365

Jeammaud A. (1982) : «Le nouveau régime du temps de travail », Droit social p. 305

Lyon Caen A. (1996), « Le maintien de l'emploi », Droit Social p. 655-659.

Mercure D, (1995), Les temporalités sociales, L'Harmattan, Paris

Morin M-L. (1998), La loi quinquennale, une étape pour le régime juridique du temps de travail », Travail et emploi, $\mathrm{n}^{\circ} 73$

Morin M.L., de Terssac G., Thoemmes J, Sublet S, (1996) : Les accords sur le temps de travail et la loi quinquennale du 20 Décembre 1993, Rapport de recherche au CGP, Paris , $180 \mathrm{p}$.

Naville P., (1972) : Temps et techniques: structure de la vie de travail, Genève, Librairie

Droz

Pélissier J, (1997) : Le droit de l'emploi, Paris, Dalloz.

Ray J.E., (1987) : «Les accords sur le temps de travail », Droit Social p. 99 -114. 
Ray J.E. (1994), Partage du travail et plan social », Droit social, no 5, pp. 444-455.

Rotschild Souriac M.-A. (1997), Engagements et arrangements sur l'emploi, quelle cité juridique, Droit social, $\mathrm{n}^{\circ} 12$, pp. 1061-1069.

Supiot A. (1995) : «Temps de travail, pour une concordance des temps », Droit social p. 947

Thoemmes J, (1997), Vers la fin du temps de travail? Thèse de Sociologie, Toulouse, Université Toulouse Le Mirail, 432 p.

Thoemmes J, de Terssac G, (1995), La construction des arrangements temporels: analyse d'accords sur 11 ans, dans une région. In Acte du colloque «Temps de travail: nouveaux enjeux, nouvelles normes, nouvelles mesures », $6^{\circ}$ Séminaire International sur le Temps de Travail, Blankenberge, Belgique, pp 190-198.

Thoemmes J, de Terssac G, (1997) La négociation du temps de travail et les composantes du référentiel temporel, Loisir et Société, vol. 20, pp 51-71 